\title{
REGULAR SEQUENCES AND THE JOINT SPECTRAL RADIUS
}

\author{
MICHAEL COONS
}

\begin{abstract}
We classify the growth of a $k$-regular sequence based on information from its $k$-kernel. In order to provide such a classification, we introduce the notion of a growth exponent for $k$-regular sequences and show that this exponent is equal to the joint spectral radius of any set of a special class of matrices determined by the $k$-kernel.
\end{abstract}

\section{INTRODUCTION}

Let $\mathbb{K}$ be a field of characteristic zero. The $k$-kernel of $f: \mathbb{Z}_{\geqslant 0} \rightarrow \mathbb{K}$ is the set

$$
\operatorname{Ker}_{k}(f):=\left\{\left\{f\left(k^{\ell} n+r\right)\right\}_{n \geqslant 0}: \ell \geqslant 0,0 \leqslant r<k^{\ell}\right\} .
$$

The sequence $f$ is called $k$-automatic provided the set $\operatorname{Ker}_{k}(f)$ is finite [6]. In 1992, as a generalisation of automatic sequences, Allouche and Shallit [1] introduced the notion of regular sequences. By their definition, a sequence $f$ taking values in $\mathbb{K}$ is called $k$-regular, for an integer $k \geqslant 1$, provided the $\mathbb{K}$-vector space $\left\langle\operatorname{Ker}_{k}(f)\right\rangle_{\mathbb{K}}$ spanned by $\operatorname{Ker}_{k}(f)$ is finite dimensional. Connecting regular sequences to finite sets of matrices, Allouche and Shallit [1, Lemma 4.1] showed that $a \mathbb{K}$ valued sequence $f$ is $k$-regular if and only if there exist a positive integer $d$, a finite set of matrices $\mathcal{A}_{f}=\left\{\mathbf{A}_{0}, \ldots, \mathbf{A}_{k-1}\right\} \subseteq \mathbb{K}^{d \times d}$, and vectors $\mathbf{v}, \mathbf{w} \in \mathbb{K}^{d}$ such that $f(n)=\mathbf{w}^{T} \mathbf{A}_{i_{0}} \cdots \mathbf{A}_{i_{s}} \mathbf{v}$, where $(n)_{k}=i_{s} \cdots i_{0}$ is the base- $k$ expansion of $n$. Moreover, their proof showed that all such collections of matrices can be described (or constructed) by considering spanning sets of $\left\langle\operatorname{Ker}_{k}(f)\right\rangle_{\mathbb{K}}$.

In their seminal paper, Allouche and Shallit [1, Theorem 2.10] proved that given a $k$-regular sequence $f$, there is a positive constant $c_{f}$ such that $f(n)=O\left(n^{c_{f}}\right)$.

In this paper, we determine the optimal value of the constant $c_{f}$. To state our result, we require a few definitions. Let $k \geqslant 1$ be an integer and $f: \mathbb{Z}_{\geqslant 0} \rightarrow \mathbb{K}$ be a (not eventually zero) $k$-regular sequence. We define the growth exponent of $f$, denoted $\operatorname{GrExp}(f)$, by

$$
\operatorname{GrExp}(f):=\limsup _{\substack{n \rightarrow \infty \\ f(n) \neq 0}} \frac{\log |f(n)|}{\log n}
$$

The joint spectral radius of a finite set of matrices $\mathcal{A}=\left\{\mathbf{A}_{0}, \mathbf{A}_{1}, \ldots, \mathbf{A}_{k-1}\right\}$, denoted $\rho(\mathcal{A})$, is defined as the real number

$$
\rho(\mathcal{A})=\limsup _{n \rightarrow \infty} \max _{0 \leqslant i_{0}, i_{1}, \ldots, i_{n-1} \leqslant k-1}\left\|\mathbf{A}_{i_{0}} \mathbf{A}_{i_{1}} \cdots \mathbf{A}_{i_{n-1}}\right\|^{1 / n},
$$

where $\|\cdot\|$ is any (submultiplicative) matrix norm. This quantity was introduced by Rota and Strang 8 and has a wide range of applications. For an extensive treatment, see Jungers's monograph 7 .

Theorem 1. Let $k \geqslant 1$ and $d \geqslant 1$ be integers and $f: \mathbb{Z}_{\geqslant 0} \rightarrow \mathbb{K}$ be a not eventually zero) $k$-regular sequence. If $\mathcal{A}_{f}$ is any collection of $k$ integer matrices associated to a basis of the $\mathbb{K}$-vector space $\left\langle\operatorname{Ker}_{k}(f)\right\rangle_{\mathbb{K}}$, then

$$
\log _{k} \rho\left(\mathcal{A}_{f}\right)=\operatorname{GrExp}(f),
$$

where $\log _{k}$ denotes the base- $k$ logarithm.

Date: February 1, 2018.

The research of M. Coons was supported by ARC grant DE140100223. 
We note that Theorem 1 holds for $\mathbb{K}$ replaced by any Nötherian ring $R$, where $\mathcal{A}_{f}$ is any collection of $k$ matrices associated to an $R$-module basis of the $R$-module spanned by $\operatorname{Ker}_{k}(f)$, where this $R$-module is viewed as an $R$-submodule of the set of a sequences with entries in $R$. In particular, the result holds for the ring $\mathbb{Z}$.

Remark 2. In engineering circles, for certain choices of $\mathcal{A}$ related to a set $D$ of forbidden sign patterns, the quantity $\log _{2} \rho(\mathcal{A})$ is sometimes referred to as the capacity of the set $D$, denoted $\operatorname{cap}(D)$. See Jungers, Blondel, and Protasov [4, Section II] for details.

\section{The Growth EXPonent of a REgular SEQUEnCE}

In this section, all matrices are assumed to have entries in $\mathbb{K}$ and all regular sequences are supposed to not eventually be zero.

Lemma 3. Let $k \geqslant 1$ be an integer and $\mathcal{A}=\left\{\mathbf{A}_{0}, \mathbf{A}_{1}, \ldots, \mathbf{A}_{k-1}\right\}$ be a finite set of matrices. Given $\varepsilon>0$ then there is a submultiplicative matrix norm $\|\cdot\|$ such that $\left\|\mathbf{A}_{i}\right\|<\rho(\mathcal{A})+\varepsilon$ for each $i \in\{0,1, \ldots, k-1\}$.

Lemma 3 can be found in Blondel et al. [5, Proposition 4], though it was first given in the original paper of Rota and Strang [8].

Proposition 4. Let $k \geqslant 2$ be an integer and $f: \mathbb{Z}_{\geqslant 0} \rightarrow \mathbb{K}$ be a $k$-regular function. For any $\varepsilon>0$, there is a constant $c=c(\varepsilon)>0$ such that for all $n \geqslant 1$,

$$
\frac{|f(n)|}{n^{\log _{k}\left(\rho\left(\mathcal{A}_{f}\right)+\varepsilon\right)}} \leqslant c
$$

where $\mathcal{A}_{f}$ is the set any set of matrices associated to a spanning set of $\left\langle\operatorname{Ker}_{k}(f)\right\rangle_{\mathbb{K}}$.

Proof. Let $\varepsilon>0$ be given and let $\|\cdot\|$ be a matrix norm such that the conclusion of Lemma 3 holds. Then

$$
|f(n)| \leqslant\|\mathbf{v}\| \cdot\|\mathbf{w}\| \cdot \prod_{j=0}^{s}\left\|\mathbf{A}_{i_{j}}\right\| \leqslant\|\mathbf{v}\| \cdot\|\mathbf{w}\| \cdot(\rho(\mathcal{A})+\varepsilon)^{s},
$$

where the base- $k$ expansion of $n$ is $i_{s} \cdots i_{0}$. Using the bound $s \leqslant \log _{k} n$ with some rearrangement gives the result.

Lemma 5. Let $k \geqslant 1$ be an integer and $\mathcal{A}=\left\{\mathbf{A}_{0}, \mathbf{A}_{1}, \ldots, \mathbf{A}_{k-1}\right\}$ be a finite set of matrices. If $\varepsilon>0$ is a real number, then there is a positive integer $m$ and a matrix $\mathbf{A}_{i_{0}} \cdots \mathbf{A}_{i_{m-1}}$, such that

$$
(\rho(\mathcal{A})-\varepsilon)^{m}<\rho\left(\mathbf{A}_{i_{0}} \cdots \mathbf{A}_{i_{m-1}}\right)<(\rho(\mathcal{A})+\varepsilon)^{m} .
$$

Proof. This is a direct consequence of the definition of the joint spectral radius.

Now let $k \geqslant 2$ be an integer, and suppose that $f: \mathbb{Z}_{\geqslant 0} \rightarrow \mathbb{K}$ is an unbounded $k$-regular sequence. Given a word $w=i_{s} \cdots i_{0} \in\{0, \ldots, k-1\}^{*}$, we let $[w]_{k}$ denote the natural number such that $(n)_{k}=w$. Let $\left\{\{f(n)\}_{n \geqslant 0}=\right.$ $\left.\left\{g_{1}(n)\right\}_{n \geqslant 0}, \ldots,\left\{g_{d}(n)\right\}_{n \geqslant 0}\right\}$ be a basis for the $\mathbb{K}$-vector space $\left\langle\operatorname{Ker}_{k}(f)\right\rangle_{\mathbb{K}}$. Then for each $i \in\{0,1, \ldots, k-1\}$, the sequences $\left\{g_{1}(k n+i)\right\}_{n \geqslant 0}, \ldots,\left\{g_{d}(k n+i)\right\}_{n \geqslant 0}$ can be expressed as $\mathbb{K}$-linear combinations of $\left\{g_{1}(n)\right\}_{n \geqslant 0}, \ldots,\left\{g_{d}(n)\right\}_{n \geqslant 0}$ and hence there is a set of $d \times d$ matrices $\mathcal{A}_{f}=\left\{\mathbf{A}_{0}, \ldots, \mathbf{A}_{k-1}\right\}$ with entries in $\mathbb{K}$ such that

$$
\mathbf{A}_{i}\left[g_{1}(n), \ldots, g_{d}(n)\right]^{T}=\left[g_{1}(k n+i), \ldots, g_{d}(k n+i)\right]^{T}
$$

for $i=0, \ldots, k-1$ and all $n \geqslant 0$. In particular, if $i_{s} \cdots i_{0}$ is the base- $k$ expansion of $n$, then $\mathbf{A}_{i_{0}} \cdots \mathbf{A}_{i_{s}}\left[g_{1}(0), \ldots, g_{d}(0)\right]^{T}=\left[g_{1}(n), \ldots, g_{d}(n)\right]^{T}$. (We note that this holds even if we pad the base- $k$ expansion of $n$ with zeros at the beginning.) We call such a set of matrices $\mathcal{A}_{f}$, constructed in this way, a set of matrices associated to a basis of $\left\langle\operatorname{Ker}_{k}(f)\right\rangle_{\mathbb{K}}$. 
This construction allows us to provide a lower bound analogue of Proposition 4 .

Proposition 6. Let $k \geqslant 2$ be an integer and $f: \mathbb{Z}_{\geqslant 0} \rightarrow \mathbb{K}$ be a $k$-regular function. For any $\varepsilon>0$, there is a constant $c=c(\varepsilon)>0$ such that for infinitely many $n \geqslant 1$,

$$
\frac{|f(n)|}{n^{\log _{k}\left(\rho\left(\mathcal{A}_{f}\right)-\varepsilon\right)}} \geqslant c
$$

where $\mathcal{A}_{f}$ is any set of matrices associated to a basis of $\left\langle\operatorname{Ker}_{k}(f)\right\rangle_{\mathbb{K}}$.

Proof. Let $\varepsilon>0$ be given. Then by Lemma 5 there is a positive integer $m$ and a matrix $\mathbf{A}=\mathbf{A}_{i_{0}} \cdots \mathbf{A}_{i_{m-1}}$ such that $\rho(\mathbf{A})>\left(\rho\left(\mathcal{A}_{f}\right)-\varepsilon\right)^{m}$. Let $\lambda$ be an eigenvalue of $\mathbf{A}$ with $|\lambda|=\rho(\mathbf{A})$. Then there is an eigenvector $\mathbf{y}$ such that $\mathbf{A y}=\lambda \mathbf{y}$. Pick a vector $\mathbf{x}$ such that $\mathbf{x}^{T} \mathbf{y}=c_{1} \neq 0$. Then

$$
\left|\mathbf{x}^{T} \mathbf{A}^{n} \mathbf{y}\right|=\left|c_{1}\right| \cdot|\lambda|^{n}=\left|c_{1}\right| \cdot \rho(\mathbf{A})^{n}>\left|c_{1}\right| \cdot\left(\rho\left(\mathcal{A}_{f}\right)-\varepsilon\right)^{n m} .
$$

Using a method developed by Bell, Coons, and Hare [3], it follows (see Appendix $\mathrm{A}$ for details) that there are words $u_{1}, \ldots, u_{d}, v_{1}, \ldots, v_{t}$ from $\{0,1, \ldots, k-1\}^{*}$ and a positive constant $c_{2}$ such that for each $n \geqslant 0$ there is an element from

$$
\left\{\left|f\left(\left[u_{i}\left(i_{m-1} \cdots i_{0}\right)^{n} v_{j}\right]_{k}\right)\right|: i=1, \ldots, d, j=1, \ldots, t\right\},
$$

which is at least $c_{2}\left(\rho\left(\mathcal{A}_{f}\right)-\varepsilon\right)^{n m}$. Here, as previously, we have used the notation $[w]_{k}$ to be the integer $n$ such that $(n)_{k}=w$.

If $M=\max \left\{\left|u_{i}\right|,\left|v_{j}\right|: i=1, \ldots, d, j=1, \ldots, t\right\}$, then

$$
N=\left[u_{i}\left(i_{m-1} \cdots i_{0}\right)^{n} v_{j}\right]_{k}<k^{2 M+n m},
$$

so that $\log _{k}(N)-2 M<n m$. Thus, by the finding of the previous paragraph, there are infinitely many $N$ such that

$$
\frac{|f(N)|}{N^{\log _{k}\left(\rho\left(\mathcal{A}_{f}\right)-\varepsilon\right)}}=\frac{|f(N)|}{\left(\rho\left(\mathcal{A}_{f}\right)-\varepsilon\right)^{\log _{k} N}}>\frac{c_{2}}{\left(\rho\left(\mathcal{A}_{f}\right)-\varepsilon\right)^{2 M}},
$$

which is the desired result.

Proof of Theorem 1. For a given $\varepsilon>0$, Proposition 4 implies that

$$
\lim _{n \rightarrow \infty} \frac{|f(n)|}{n^{\log _{k}\left(\rho\left(\mathcal{A}_{f}\right)+2 \varepsilon\right)}}=0
$$

and Proposition 6 implies that

$$
\limsup _{n \rightarrow \infty} \frac{|f(n)|}{n^{\log _{k}\left(\rho\left(\mathcal{A}_{f}\right)-2 \varepsilon\right)}}=\infty
$$

Taken together these give

$$
\log _{k}\left(\rho\left(\mathcal{A}_{f}\right)-2 \varepsilon\right) \leqslant \operatorname{GrExp}(f) \leqslant \log _{k}\left(\rho\left(\mathcal{A}_{f}\right)+2 \varepsilon\right) .
$$

Since $\varepsilon$ can be taken arbitrarily small, this proves the theorem.

We end this section by highlighting one major difference between Proposition 4 and Proposition 6. Proposition 4 is true for $\mathcal{A}_{f}$ related to any spanning set of the $\mathbb{K}$-vector space $\left\langle\operatorname{Ker}_{k}(f)\right\rangle_{\mathbb{K}}$, while Proposition $\left[6\right.$ requires $\mathcal{A}_{f}$ to be associated to a basis of $\left\langle\operatorname{Ker}_{k}(f)\right\rangle_{\mathbb{K}}$. In fact, these two propositions give the following corollary.

Corollary 7. Let $k \geqslant 2$ be an integer and $f: \mathbb{Z}_{\geqslant 0} \rightarrow \mathbb{K}$ be a $k$-regular function. If $\mathcal{B}_{f}$ is any set of matrices associated to $f$ and $\mathcal{A}_{f}$ is any set of matrices associated to a basis of $\left\langle\operatorname{Ker}_{k}(f)\right\rangle_{\mathbb{K}}$, then $\rho\left(\mathcal{A}_{f}\right) \leqslant \rho\left(\mathcal{B}_{f}\right)$. 
Equality in the conclusion of the above corollary would be desirable, but unfortunately, this is not (in general) the case. To see this, consider the 2-regular function $f$, where, for $(n)_{2}=i_{s} \cdots i_{0}$, we have $f(n)=\mathbf{w}^{T} \mathbf{A}_{i_{0}} \cdots \mathbf{A}_{i_{s}} \mathbf{v}$, with

$$
\mathcal{A}_{f}=\left\{\mathbf{A}_{0}, \mathbf{A}_{1}\right\}=\left\{\left(\begin{array}{ll}
1 & 0 \\
0 & 1
\end{array}\right)\right\}, \quad \mathbf{w}^{T}=\left[\begin{array}{ll}
1 & 0
\end{array}\right], \quad \text { and } \quad \mathbf{v}=\left[\begin{array}{ll}
1 & 1
\end{array}\right]^{T} .
$$

Then also for any number $x>1$, we have $f(n)=\mathbf{x}^{T} \mathbf{B}_{i_{0}} \cdots \mathbf{B}_{i_{s}} \mathbf{y}$, with

$$
\mathcal{B}_{f}=\left\{\mathbf{B}_{0}, \mathbf{B}_{1}\right\}=\left\{\left(\begin{array}{lll}
1 & 0 & 0 \\
0 & 1 & 0 \\
0 & 0 & x
\end{array}\right)\right\}, \quad \mathbf{x}^{T}=\left[\begin{array}{lll}
1 & 0 & 0
\end{array}\right], \quad \text { and } \quad \mathbf{y}=\left[\begin{array}{lll}
1 & 1 & 0
\end{array}\right]^{T},
$$

and

$$
\rho\left(\mathcal{A}_{f}\right)=1<x=\rho\left(\mathcal{B}_{f}\right)
$$

\section{Appendix A.}

For a given $\varepsilon>0$, we had by Lemma 5 that there is a positive integer $m$ and a matrix $\mathbf{A}=\mathbf{A}_{i_{0}} \cdots \mathbf{A}_{i_{m-1}}$ such that $\rho(\mathbf{A})>\left(\rho\left(\mathcal{A}_{f}\right)-\varepsilon\right)^{m}$. Choosing an eigenvalue $\lambda$ of $\mathbf{A}$ with $|\lambda|=\rho(\mathbf{A})$, we found vectors $\mathbf{x}$ and $\mathbf{y}$ such that $\mathbf{x}^{T} \mathbf{y}=c_{1} \neq 0$ and

$$
\left|\mathbf{x}^{T} \mathbf{A}^{n} \mathbf{y}\right|=\left|c_{1}\right| \cdot|\lambda|^{n}=\left|c_{1}\right| \cdot \rho(\mathbf{A})^{n}>\left|c_{1}\right| \cdot\left(\rho\left(\mathcal{A}_{f}\right)-\varepsilon\right)^{n m} .
$$

In this appendix, we follow an argument of Bell, Coons, and Hare [3, p. 198] to provide the existence of words $u_{1}, \ldots, u_{d}, v_{1}, \ldots, v_{t}$ from $\{0,1, \ldots, k-1\}^{*}$ such that for each $n \geqslant 0$ there is an element from

$$
\left\{\left|f\left(\left[u_{i}\left(i_{m-1} \cdots i_{0}\right)^{n} v_{j}\right]_{k}\right)\right|: i=1, \ldots, d, j=1, \ldots, t\right\},
$$

which is at least $c_{2}\left(\rho\left(\mathcal{A}_{f}\right)-\varepsilon\right)^{n m}$.

To this end, let $k \geqslant 2$ be an integer, suppose that $f: \mathbb{Z}_{\geqslant 0} \rightarrow \mathbb{K}$ is an unbounded $k$-regular sequence, and $\mathcal{A}_{f}=\left\{\mathbf{A}_{0}, \ldots, \mathbf{A}_{k-1}\right\}$ be a set of matrices associated to a basis $\left\{\{f(n)\}_{n \geqslant 0}=\left\{g_{1}(n)\right\}_{n \geqslant 0}, \ldots,\left\{g_{d}(n)\right\}_{n \geqslant 0}\right\}$ of the $\mathbb{K}$-vector space $\left\langle\operatorname{Ker}_{k}(f)\right\rangle_{\mathbb{K}}$.

We claim that the $\mathbb{K}$-span of the vectors $\left[g_{1}(i), \ldots, g_{d}(i)\right]$, as $i$ ranges over all natural numbers, must span all of $\mathbb{K}^{d}$. If this were not the case, then their span would be a proper subspace of $\mathbb{K}^{d}$ and hence the span would have a non-trivial orthogonal complement. In particular, there would exist $c_{1}, \ldots, c_{d} \in \mathbb{K}$, not all zero, such that

$$
c_{1} g_{1}(n)+\cdots+c_{d} g_{d}(n)=0
$$

for every $n$, contradicting the fact that $g_{1}(n), \ldots, g_{d}(n)$ are $\mathbb{K}$-linearly independent sequences.

Let $\left\langle\mathcal{A}_{f}\right\rangle$ denote the semigroup generated by the elements of $\mathcal{A}_{f}$. We have just shown that there exist words $\mathbf{X}_{1}, \ldots, \mathbf{X}_{d}$ in $\left\langle\mathcal{A}_{f}\right\rangle$ such that

$$
\left[g_{1}(0), \ldots, g_{d}(0)\right] \mathbf{X}_{1}, \ldots,\left[g_{1}(0), \ldots, g_{d}(0)\right] \mathbf{X}_{d}
$$

$\operatorname{span} \mathbb{K}^{d}$

Now consider $\mathbf{x}^{T} \mathbf{A}^{n} \mathbf{y}$ as described in the first paragraph of this appendix. By construction, we may write $\mathbf{x}^{T}=\sum_{j} \alpha_{j}\left[g_{1}(0), \ldots, g_{d}(0)\right] \mathbf{X}_{j}$ for some complex numbers $\alpha_{j}$. Then

$$
\mathbf{x}^{T} \mathbf{A}^{n}=\sum_{j} \alpha_{j}\left[g_{1}(0), \ldots, g_{d}(0)\right] \mathbf{X}_{j} \mathbf{A}^{n} .
$$

Let $u_{j}$ be the word in $\{0,1, \ldots, k-1\}^{*}$ corresponding to $\mathbf{X}_{j}$ and let $y=i_{s} \cdots i_{0}$ be the word in $\{0, \ldots, k-1\}^{*}$ corresponding to $\mathbf{A}$; that is $y=i_{s} \cdots i_{0}$ where $\mathbf{A}=\mathbf{A}_{i_{s}} \cdots \mathbf{A}_{i_{0}}$ and similarly for $u_{j}$. Then we have

$$
\left[g_{1}(0), \ldots, g_{d}(0)\right] \mathbf{X}_{j} \mathbf{A}^{n}=\left[g_{1}\left(\left[u_{j} y^{n}\right]_{k}\right), \ldots, g_{d}\left(\left[u_{j} y^{n}\right]_{k}\right)\right]^{T} \text {. }
$$


Write $\mathbf{y}^{T}=\left[\beta_{1}, \ldots, \beta_{d}\right]$. Then

$$
\mathbf{x}^{T} \mathbf{A}^{n} \mathbf{y}=\sum_{i, j} \alpha_{i} \beta_{j} g_{j}\left(\left[u_{i} y^{n}\right]_{k}\right) .
$$

By assumption, each of $\left\{g_{1}(n)\right\}_{n \geqslant 0}, \ldots,\left\{g_{d}(n)\right\}_{n \geqslant 0}$ is in the $\mathbb{K}$-vector space generated by $\operatorname{Ker}_{k}(f)$, and hence there exist natural numbers $p_{1}, \ldots, p_{t}$ and $q_{1}, \ldots, q_{t}$ with $0 \leqslant q_{m}<k^{p_{m}}$ for $m=1, \ldots, t$ such that each of for $s=1, \ldots, d$, we have $g_{s}(n)=\sum_{i=1}^{t} \gamma_{i, s} f\left(k^{p_{i}} n+q_{i}\right)$ for some constants $\gamma_{i, s} \in \mathbb{K}$. Then

$$
\mathbf{x}^{T} \mathbf{A}^{n} \mathbf{y}=\sum_{i, j, \ell} \alpha_{i} \beta_{j} \gamma_{\ell, j} f\left(\left[u_{i} y^{n} v_{\ell}\right]_{k}\right),
$$

where $v_{\ell}$ is the unique word in $\{0,1, \ldots, k-1\}^{*}$ of length $p_{\ell}$ such that $\left[v_{\ell}\right]_{k}=q_{\ell}$. Let $K=\sum_{i, j, \ell}\left|\alpha_{i}\right| \cdot\left|\beta_{j}\right| \cdot\left|\gamma_{\ell, j}\right|$. Then since $\left|\mathbf{x}^{T} \mathbf{A}^{n} \mathbf{y}\right| \geqslant\left|c_{1}\right| \cdot\left(\rho\left(\mathcal{A}_{f}\right)-\varepsilon\right)^{n m}$ for all $n$, some element from

$$
\left.\left\{\left|f\left(\left[u_{i} y^{n} v_{j}\right]_{k}\right)\right|: i=1, \ldots, d, j=1, \ldots, t\right\}\right\}
$$

is at least $\left(\left|c_{1}\right| / K\right) \cdot\left(\rho\left(\mathcal{A}_{f}\right)-\varepsilon\right)^{n m}$ for each $n$.

Acknowledgements. We thank Björn Rüffer for several useful conversations.

\section{REFERENCES}

1. Jean-Paul Allouche and Jeffrey Shallit, The ring of $k$-regular sequences, Theoret. Comput. Sci. 98 (1992), no. 2, 163-197. MR 1166363 (94c:11021)

2. Jason P. Bell, Michael Coons, and Kevin G. Hare, Growth degree classification for finitely generated semigroups of integer matrices, Semigroup Forum, to appear.

3. W The minimal growth of a k-regular sequence, Bull. Aust. Math. Soc. 90 (2014), no. 2, 195-203. MR 3252000

4. Vincent D. Blondel, Raphaël Jungers, and Vladimir Protasov, On the complexity of computing the capacity of codes that avoid forbidden difference patterns, IEEE Trans. Inform. Theory $\mathbf{5 2}$ (2006), no. 11, 5122-5127. MR 2300380 (2007m:94086)

5. Vincent D. Blondel, Yurii Nesterov, and Jacques Theys, On the accuracy of the ellipsoid norm approximation of the joint spectral radius, Linear Algebra Appl. 394 (2005), 91-107. MR 2100578 (2005i:15043)

6. Alan Cobham, Uniform tag sequences, Math. Systems Theory 6 (1972), 164-192. MR 0457011 (56 \#15230)

7. Raphaël Jungers, The joint spectral radius, Lecture Notes in Control and Information Sciences, vol. 385, Springer-Verlag, Berlin, 2009, Theory and applications. MR 2507938 (2011c:15001)

8. Gian-Carlo Rota and Gilbert Strang, A note on the joint spectral radius, Nederl. Akad. Wetensch. Proc. Ser. A 63 = Indag. Math. 22 (1960), 379-381. MR 0147922 (26 \#5434)

School of Math. and Phys. Sciences, University of Newcastle, Callaghan, Australia E-mail address: Michael.Coons@newcastle.edu.au 